\title{
A simple model for the saturation of coronal X-ray emission of rapidly rotating late-type stars
}

\author{
R. D. Ryan ${ }^{1,2}$, T. Neukirch ${ }^{1}$, and M. Jardine ${ }^{3}$ \\ ${ }^{1}$ School of Mathematics \& Statistics, University of St. Andrews, St. Andrews KY16 9SS, UK \\ e-mail: thomas@mcs.st-and.ac.uk \\ 2 Observatoire de Strasbourg, 11 rue de l'Université, Strasbourg 67000, France \\ 3 School of Physics \& Astronomy, University of St. Andrews, St. Andrews KY16 9SS, UK
}

Received 14 January 2004 / Accepted 7 December 2004

\begin{abstract}
An explanation of the observed saturation of X-ray emission with increasing rotation rate of late-type stars is suggested. The suggestion is based on a simple model for the closed field line region of the magnetized stellar corona (stellar magnetosphere). The model assumes a magnetic dipole field aligned with the rotation axis confining a co-rotating, isothermal plasma. The effects of a stellar wind and of toroidal fields are neglected in the model. The closed field line region in the model is defined by those field lines on which the ratio of plasma pressure to magnetic pressure (plasma $\beta$ ) does not exceed one. Using scaling relations of surface magnetic field strength, surface density and coronal temperature with angular velocity, the model is used to calculate the stellar X-ray emission measure as a function of rotation rate. The results are in good agreement with the saturation in X-ray emission with rotation that is observed in G-K stars. The dependence of the results on the parameters of the model will be discussed.
\end{abstract}

Key words. magnetohydrodynamics (MHD) - magnetic fields - stars: coronae - stars: late-type - stars: activity

\section{Introduction}

For rapidly rotating single stars in open clusters, a correlation between magnetic activity and the stellar rotation rate has been well established (e.g. Noyes et al. 1984), with the rotation rate being a crucial parameter of this activity in stars of a given spectral type (Kraft 1967; Durney 1972). This correlation can be understood in terms of the generation of large scale flux from a dynamo, the efficiency of which will itself depend upon the rotation of the fluid (Parker 1955, 1979). The magnetic field couples the sub-photospheric convection zone with the stellar chromosphere, transition region and corona, allowing convective turbulent kinetic energy to be converted into excess magnetic energy which may then be dissipated, thereby allowing the maintenance of a hot stellar wind which acts to brake the stellar rotation (see Mestel \& Spruit 1987; Collier Cameron et al. 1991). This leads to a subsequent decrease in the magnetic flux maintained by the dynamo and the associated coronal magnetic activity that is observed in the optical through to the $\mathrm{X}$-ray spectrum bands.

Other measurable indicators of stellar magnetic activity include the surface starspot coverage and the emission from the chromosphere, transition region or the corona. One of the best correlations of magnetic activity with rotation is often obtained when magnetic activity is measured as a function of $P / \tau_{\mathrm{c}}$ where $P$ is the rotation period and $\tau_{\mathrm{c}}$ is the convective turnover time at the base of the stars convective envelope. All of these indicators show an increase with rotation rate, followed by saturation (Noyes et al. 1984; Hempelmann et al. 1995; Stauffer et al. 1997; Pizzolato et al. 2003) with recent evidence also showing indications of a supersaturation phenomenon where the X-ray emission decreases with increasing rotation rate, after the saturation plateau (e.g. Prosser et al. 1996; Randich 1998).

A number of different explanations have been invoked in order to explain this saturation phenomenon. Principal amongst them has been that of saturation of the dynamo mechanism. The generation of very strong magnetic fields by dynamo processes may be limited by the Lorentz back-reaction on the fluid motions with shear stressing across field boundaries (e.g. Charbonneau \& MacGregor 1992). If the magnetic activity indicators are directly related to the strength of the stellar magnetic field this would imply a saturation of the activity indicators due to a saturation of the magnetic field generation process. A more extended discussion of possible relations between activity saturation and candidate mechanisms (in terms of nonlinear dynamo theory) that could explain this behaviour, is given by Rosner (2000).

Alternatively, if the dynamo process itself does not saturate the relationship between magnetic field strength and magnetic activity indicators could change. One example of this has been proposed by Vilhu (1984). The author assumes that the activity 
indicators are associated with the magnetically active regions on the stellar surface. If the area covered by active regions increases with the increasing average magnetic field strength, saturation sets in at the rotation rate where the complete surface of a star is covered by active regions. For stars rotating faster than this critical rotation rate, the magnetic field strength would increase whereas the emission would not.

Another possibility to consider is that the heating mechanism itself could saturate. This may occur either because the driving motions are being suppressed by the stronger fields or because the maximum available power for the heating has already been fully exploited.

However, a number of workers have advanced the idea that it may not be necessary to invoke saturation of the dynamo and that the observed saturation in activity may be reproduced or mimicked through other means. One such argument was advanced by Solanki et al. (1997) where the authors suggest that increasing rotation leads to a concentration of magnetic flux near the poles of solar-type stars. In their models, buoyant magnetic flux tubes are deflected by the Coriolis force to emerge at higher latitudes (Schüssler \& Solanki 1992; Schüssler et al. 1996). Upon emergence, they exert a reduced braking torque compared to low latitude flux tubes and the extra open-field line regions will act to reduce the trapping and heating of upper atmospheric plasma. Together with the results of O'Dell et al. (1995) this offers an argument against dynamo saturation at low rotation rates and mimics the observed saturation of star-spot activity (which acts as a tracer for the total surface magnetic flux in these models).

The models of Jardine \& Unruh (1999) propose an alternative "centrifugal stripping" model which reproduces the observed saturation in X-ray emission and either mimics the dynamo saturation or disguises the rotation rate at which the saturation sets in. In their models, increasing rotation leads to a rise in the pressure and density in the outer parts of the largest magnetic loops. These stresses may, in turn, cause previously closed field lines to break open (as originally suggested by Mestel \& Spruit 1987) provided that the centrifugal forces are sufficient to overcome the magnetic forces that confine the plasma and this leads to a reduction in the extent of the closedfield corona. In these models, it is the co-rotation radius that marks the heights (above the stellar photosphere) at which the centrifugal stressing begins and which are more susceptible to the onset of radiative instabilities due to the increased plasma density.

At present it is unclear which of the discussed mechanisms is actually causing the saturation effects (e.g. Pizzolato et al. 2003). In principle there could be several mechanisms at work in parallel. If the dynamo process itself saturates one would expect that the surface magnetic field of the stars is also subject to a saturation effect. However, O'Dell et al. (1995) argue that there is no apparent saturation of starspot coverage, and Saar (2001) remarks that saturation, if present, may well be restricted to the plage component alone. Also, Mathioudakis et al. (1995) demonstrate that different indicators of magnetic activity appear to saturate at different angular velocities.

We therefore think that it is worthwhile to investigate the effects of mechanisms not depending on a saturation of the dynamo. The purpose of this paper is to demonstrate that both saturation and supersaturation of the X-ray emission do not necessarily indicate saturation of the dynamo mechanism and we aim to reproduce this saturation phenomenon by the construction of a relatively simple (but nonetheless physically plausible) MHD model. We would like to emphasize that we do not intend to imply that there is no saturation of the dynamo mechanism at all, but rather that there are other mechanisms which may mimic this phenomenon and mask the rotation rate at which saturation will appear. However, to be able to identify the consequences of our model we will make the assumption that there is no dynamo saturation at all. In principle, dynamo saturation could be included in a future version of our model, but we have refrained from including it in the present work as this would only obscure our main point.

In the first part of this paper (Sect. 2) we outline an ideal MHD formulation of the coronae of rapidly rotating late-type stars and introduce a cut-off criterion to allow us to calculate emission measures over a finite magnetospheric volume. The resulting equations can be solved by standard numerical methods. The results of these calculations are presented and discussed in the Sect. 3, and the conclusions are given in Sect. 4. Two appendices are included, in order to detail some of our analytical derivations.

\section{The model}

\subsection{MHD formulation}

In the present paper we will consider single late-type stars (of spectral class $\mathrm{F}$ to $\mathrm{K}$ ). We simplify our model by assuming that the magnetic fields of these stars are axisymmetric with the axis of symmetry being aligned with the rotation axis of the star (assumed to be the $z$-axis). Using spherical coordinates $r$, $\theta, \phi$ and ignoring any azimuthal component, we can express the magnetic field as

$\boldsymbol{B}=\frac{1}{r \sin \theta} \nabla A \times \boldsymbol{e}_{\phi}$

The flux function $A$ is defined by

$A=r \sin \theta A_{\phi}$,

where $A_{\phi}$ is the toroidal component of the magnetic vector potential. Lines of constant $A$ are magnetic field lines in this case (if a $\phi$-component would be included they would be poloidal projections of field lines).

In our model we assume that the X-ray emission originates exclusively from closed field line regions. This assumption is based on the fact that for the Sun closed field line regions are bright in X-rays, whereas regions with open field lines (coronal holes) are dark in X-rays. We therefore confine our attention to closed field line regions, and neglect poloidal outflows altogether for our model. The plasma on the closed field lines will be assumed to carry out only toroidal motion, i.e.

$\boldsymbol{v}=v_{\phi} \boldsymbol{e}_{\phi}=\Omega r \sin \theta \boldsymbol{e}_{\phi}$.

Under normal coronal conditions the plasma is well described by ideal MHD and therefore the frozen-in condition applies. 
We assume that the plasma at the coronal base is rigidly rotating with the angular velocity of the $\operatorname{star}(\Omega)$. We neglect further complications that could arise from differential rotation and the toroidal shearing of field line foot-points. Donati \& Collier Cameron (1997) determined the rate of surface shear due to differential rotation on the K0 dwarf AB Doradus to be very similar to that of the Sun. On the Sun significant shearing of loop foot-points is found to occur on timescales of weeks to months, and we believe that this justifies our assumption. The plasma on the closed field lines will thus be co-rotating with the star at angular velocity $\Omega$.

Under these assumptions the balance of forces acting on the coronal plasma is given by

$\boldsymbol{j} \times \boldsymbol{B}-\nabla p-\rho \nabla U=\mathbf{0}$,

where $\boldsymbol{j}$ is the current density given by Ampère's law $(\nabla \times \boldsymbol{B}=$ $\left.\mu_{0} \boldsymbol{j}\right), \rho$ and $p$ are the plasma density and pressure and the effective gravity acting on the plasma is given by the gradient of the effective potential

$U=-\left(\frac{G M_{\star}}{r}-\frac{G M_{\star}}{R_{\star}}\right)-\frac{1}{2} \Omega^{2}\left(r^{2} \sin ^{2} \theta-R_{\star}^{2}\right)$.

Here $U$ has been gauged to be zero at the stellar equator $\left(r=R_{\star}, \theta=\pi / 2\right)$. The effective potential ( $U$ ) given by Eq. (5) combines the contributions of both gravitational and centrifugal force.

Since the Lorentz force only acts perpendicularly to the magnetic field, the plasma pressure gradients along the field are balanced by the field aligned component of the gradient of the effective gravitational potential. Using Eq. (1) to calculate the current density from Ampère's law and to substitute for $\boldsymbol{B}$, we obtain

$\left[-\frac{1}{\mu_{0}} \nabla \cdot\left(\frac{1}{r^{2} \sin ^{2} \theta} \nabla A\right)\right] \nabla A-\nabla p-\rho \nabla U=\mathbf{0}$.

Due to our assumption of axisymmetry the pressure gradient will only have non-vanishing components in the poloidal plane. Apart from sets of measure zero $\nabla A$ and $\nabla U$ will be linearly independent vector fields in the poloidal plane and therefore we can rewrite $p$ as a function of $A$ and $U$. The gradient of $p$ takes the form

$\nabla p=\left(\frac{\partial p}{\partial A}\right)_{U} \nabla A+\left(\frac{\partial p}{\partial U}\right)_{A} \nabla U$,

where the subscripts $A$ and $U$ indicate that the partial derivatives have to be taken at constant $A$ and $U$, respectively.

Using Eq. (7) in Eq. (6) we can split Eq. (6) into a component along $\nabla A$ and a component along $\nabla U$ and get

$$
\begin{aligned}
-\nabla \cdot\left(\frac{1}{r^{2} \sin ^{2} \theta} \nabla A\right) & =\mu_{0} r^{2} \sin ^{2} \theta\left(\frac{\partial p}{\partial A}\right)_{U}, \\
\left(\frac{\partial p}{\partial U}\right)_{A} & =-\rho .
\end{aligned}
$$

Equation (8) is the $\nabla A$ component of Eqs. (6) and (9) is the $\nabla U$ component of Eq. (6).

\subsection{Plasma pressure and density}

In the present paper we will assume that the corona on closed field lines is an ideal, isothermal gas, i.e.

$p=R T \rho=\frac{k_{\mathrm{B}}}{\bar{m}} T \rho=c_{\mathrm{s}}^{2} \rho$

where $T$ is the constant temperature, $R$ is the universal gas constant, $k_{\mathrm{B}}$ the Boltzmann constant, $\bar{m}$ the mean atomic weight of the plasma and $c_{\mathrm{s}}$ the sound speed. The assumption of constant temperature, i.e. isothermal coronal plasma, has been made to keep the model as simple as possible and to avoid the introduction of additional free parameters. The constant temperature can be interpreted as an average coronal temperature.

By using Eq. (10) to express $\rho$ in terms of $p$ and substituting this expression into Eq. (9), we can derive an expression for the pressure as a function of $A$ and $U$ of the form

$p(A, U)=p_{0}(A) \exp \left(-\bar{m} U / k_{\mathrm{B}} T\right)$.

The function $p_{0}(A)$ is a free, but positive function which determines how the pressure varies from field line to field line. This variation is modulated by the $\theta$ dependence of $U$ which is due to the centrifugal force. $p_{0}(A)$ also determines the structure of the electric current density in the coronal plasma.

To complete our model we have to specify $p_{0}(A)$. Unfortunately, an MHD formulation does not include a theoretical basis or any other indication which might allow us to make our choice for $p_{0}(A)$. We have therefore used Vlasov theory as a guideline to choose a particular form of $p_{0}(A)$, following and extending previous work by Neukirch (1993). Details of this calculation can be found in Appendix A. The basic assumption is to use particle distribution functions which are drifting Maxwell distributions in velocity space, which is appropriate for an isothermal plasma. The resulting $p_{0}(A)$ has the form

$p_{0} \propto \exp \left(A / A_{0}\right)$

where $A_{0}$ is a constant.

\subsection{Potential field approximation}

Having determined the form of $p(A, U)$ (up to multiplicative constants), the mathematically rigorous way to determine model solutions would be to solve Eq. (8) for $A$ under given boundary conditions. However, in the present paper we will assume that the large scale cross-field current density associated with $(\partial p / \partial A)_{U}$ is small and therefore the deviation of the field from a potential field is also small. A similar procedure has been used in Mestel \& Spruit (1987). Mathematically this could be regarded as an expansion of $A$ in terms of a small parameter representing the strength of the coronal current density. In such an expansion we retain only the lowest order term.

In this case the right hand side of Eq. (8) vanishes and the solutions will be potential fields. In this paper we assume the resulting magnetic field of the star is a dipole field with

$A=\alpha \frac{\sin ^{2} \theta}{r}$ 
where $\alpha$ is related to the dipole moment of the star, $M_{z \star}$, by $\alpha=$ $\mu_{0} M_{z \star} / 4 \pi$ ( $\mu_{0}$ is the vacuum permeability). The corresponding magnetic field, $\boldsymbol{B}$, is given by Eq. (1) as

$\boldsymbol{B}=\alpha\left(\frac{2 \cos \theta}{r^{3}} \boldsymbol{e}_{r}+\frac{\sin \theta}{r^{3}} \boldsymbol{e}_{\theta}\right)$.

In the following we will normalise $r$ by the stellar radius $R_{\star}$ and $A$ by $\alpha / R_{\star}$ so that at the stellar equator $A$ has the value 1 . Within our approximation the coronal plasma pressure can be calculated by substituting $A$ from Eq. (13) into the general expression for the pressure, $p(A, U)$. Before we do this we introduce the dimensionless parameters

$\xi_{1}=\frac{G M_{\star} \bar{m}}{R_{\star} k_{\mathrm{B}} T_{\star}}$,

$\xi_{2}=\frac{\bar{m} \Omega^{2} R_{\star}^{2}}{k_{\mathrm{B}} T_{\star}}$,

$\xi_{3}=\frac{\alpha}{A_{0} R_{\star}}$

which allow us to rewrite $U / k_{\mathrm{B}} T$ as

$\frac{U}{k_{\mathrm{B}} T}=-\xi_{1}\left(\frac{1}{r}-1\right)-\frac{1}{2} \xi_{2}\left(r^{2} \sin ^{2} \theta-1\right)$.

If we denote the pressure of the coronal plasma at the stellar equator $(r=1, \theta=\pi / 2)$ by $p_{\star}$ and substitute we finally obtain

$$
\begin{aligned}
p= & p_{\star} \exp \left[\xi_{3}\left(\frac{\sin ^{2} \theta}{r}-1\right)\right. \\
& \left.+\xi_{1}\left(\frac{1}{r}-1\right)+\frac{1}{2} \xi_{2}\left(r^{2} \sin ^{2} \theta-1\right)\right] .
\end{aligned}
$$

The density $\rho$ which we will need later on to calculate the emission measure of the corona can be determined from $p$ using Eq. (10). Apart from a multiplicative constant it has the same spatial variation as $p$ for an isothermal atmosphere.

\subsection{Scaling assumptions}

In a similar manner to that followed by Jardine \& Unruh (1999) we will assume that the magnetic field strength and the plasma density at the coronal base $(r=1)$ as well as the coronal temperature vary with the stellar angular velocity, $\Omega$, in the form of power laws. Due to our assumption of an isothermal ideal gas this also fixes the variation of the pressure at the coronal base with angular velocity. The scaling laws have the form

$$
\begin{aligned}
& T_{\star}=T_{\odot}\left(\frac{\Omega}{\Omega_{\odot}}\right)^{n}, \\
& \rho_{\star}=\rho_{\odot}\left(\frac{\Omega}{\Omega_{\odot}}\right)^{m}, \\
& B_{\star}=B_{\odot}\left(\frac{\Omega}{\Omega_{\odot}}\right)^{q} .
\end{aligned}
$$

The subscript $\odot$ indicates solar values which will be taken from Zombeck (1990). Given these scaling laws for $T$ and $\rho_{\star}$ the coronal base pressure scales as

$$
p_{\star}=\frac{k_{\mathrm{B}} T_{\odot} \rho_{\odot}}{\bar{m}}\left(\frac{\Omega}{\Omega_{\odot}}\right)^{n+m} \text {. }
$$

At this point we would like to emphasize that these scaling laws are chosen as a mathematically simple representation of the behaviour of the physical quantities $T_{\star}, \rho_{\star}$, and $B_{\star}$ over a wide range of angular velocities, based either upon the model assumptions or the observations. These simple scaling laws allow us to undertake a systematic study the effect of different scalings on the model results, without increasing the number of parameters even further. From a formal mathematical point of view one could argue that Eqs. (20)-(22) imply that the coronal base parameters increase without bound as the angular velocity increases, and that this is certainly unphysical. However, from a physical point of view there is of course a natural upper limit for the angular velocity at which the centrifugal forces would destroy the star (roughly $230 \Omega_{\odot}$ for a star of solar mass and radius) and therefore this formal argument is not valid.

The scaling laws can be used to determine how the parameters $\xi_{1}$ and $\xi_{2}$ depend on $\Omega$

$$
\begin{aligned}
& \xi_{1}=\frac{G M_{\odot} \bar{m}}{R_{\odot} k_{\mathrm{B}} T_{\odot}} \frac{M_{\star}}{M_{\odot}} \frac{R_{\odot}}{R_{\star}}\left(\frac{\Omega}{\Omega_{\odot}}\right)^{-n} \\
& \xi_{2}=\frac{\bar{m} \Omega_{\odot}^{2} R_{\odot}^{2}}{k_{\mathrm{B}} T_{\odot}} \frac{R_{\star}^{2}}{R_{\odot}^{2}}\left(\frac{\Omega}{\Omega_{\odot}}\right)^{2-n} .
\end{aligned}
$$

This scaling of the parameters influences the amount of closed magnetic flux in our models (see Sect. 2.5). Furthermore, the scaling also influences the value of the emission measure proxy (Sect. 2.6) directly through the scaling of the coronal base density.

\subsection{The closed flux criterion}

So far, we have not made a difference between regions of closed and open flux. Rigorously speaking the magnetic dipole field which we use as a first approximation in this paper does not have any open field lines. However, following the same arguments put forward by Mestel \& Spruit (1987), the magnetic structure of the closed field line region should be reasonably well approximated by a dipole field whereas the exact structure of the open flux region does not enter the calculation of the emission measure at all.

The question then is how to determine the closed field line region within our model? In the models of Jardine \& Unruh (1999) the authors considered the position of the corotation radius $\left(r_{\text {cor }}=\left(G M_{\star} / \Omega^{2}\right)^{\frac{1}{3}}\right)$ to be the point where the corona begins to be significantly distorted by centrifugal forces and therefore take the X-ray emission to emanate solely from within the volume bound by the co-rotation radius.

This, however, neglects the confining effect of the magnetic field beyond the co-rotation radius for fast rotating stars. Also, for slow rotators the co-rotation radius will hardly indicate the correct extent of the closed field line region since thermal pressure more than the centrifugal force will be the physical quantity determining the extent of the closed field line region. Therefore we determine the extent of the closed field line regions within our model by using a heuristic criterion based on the ratio of plasma pressure to magnetic pressure. We emphasize that by having integrated the force balance equation to obtain the pressure, we have already included the effects of 
gravitational and centrifugal force in the form of the pressure given by Eq. (19).

Using the criterion specified below we calculate a cut-off radius (denoted by $r_{\mathrm{c}}$ ) in the equatorial plane. Using Eq. (13) this cut-off radius can be associated with a value of the magnetic flux function $A_{\mathrm{c}}=1 / r_{\mathrm{c}}$ in the equatorial plane. Since lines of constant $A$ are magnetic field lines we take the closed flux region to be the region between the cut-off field line and the stellar surface. Beyond this field line the flux is considered to be open.

We choose our cut-off criterion to be that point in the corona (equatorial plane) where the plasma pressure equals the magnetic pressure, i.e. where the plasma $\beta$ is equal one. In the equatorial plane $(\theta=\pi / 2)$ we have

$\frac{1}{r_{c}^{6}}=\frac{2 \mu_{0} p_{\star}}{B_{\star}^{2}} \exp \left[\left(\xi_{1}+\xi_{3}\right)\left(\frac{1}{r_{c}}-1\right)+\frac{1}{2} \xi_{2}\left(r_{c}^{2}-1\right)\right]$,

the solution of which identifies the location of the cut-off field line at a position $r_{\mathrm{c}}$. We solve Eq. (26) with a standard numerical routine which uses a globally convergent Broyden's method.

The solutions to Eq. (26) depend on $\Omega$ because the parameters $\xi_{1}$ and $\xi_{2}$ depend on $\Omega$ through the scaling relations (20), (21), and (22) and because

$\frac{2 \mu_{0} p_{\star}}{B_{\star}^{2}}=\frac{2 \mu_{0} p_{\odot}}{B_{\odot}^{2}}\left(\frac{\Omega}{\Omega_{\odot}}\right)^{n+m-2 q}=\beta_{\odot}\left(\frac{\Omega}{\Omega_{\odot}}\right)^{n+m-2 q}$,

where $\beta_{\odot}=2 \mu_{0} p_{\odot} / B_{\odot}^{2}$ is the average plasma $\beta$ value for the coronal base of a star with solar rotation rate.

Due to the strong surface magnetic field the plasma $\beta$ at the coronal base will be smaller than one, but the magnetic pressure falls off with radius much quicker than the pressure which actually decreases first but increases again for large $r$ due to the centrifugal force. Therefore the model plasma $\beta$ increases with distance from the star and eventually becomes larger than one. An illustrative example is shown in Fig. 1. The figure shows the radial dependence of the plasma $\beta$ in the equatorial plane $(\theta=\pi / 2)$ for two different angular velocities, namely $\Omega=\Omega_{\odot}$ and $\Omega=50 \Omega_{\odot}$. The other parameter values used for this example are $\beta_{\odot}=0.02, m=0.0, n=0.75, q=0.75$ and $\xi_{3}=1.0$ and a star of solar mass and radius. The cut-off radius is determined by the intersection of the plasma $\beta$ curves with the straight line $\beta=1.0$. Whereas for the case of slow rotation the cut-off radius (at about $4.7 R_{\star}$ ) much smaller than the corotation radius (at about $37 R_{\star}$ in this case), one can see that the cut-off radius $\left(3.6 R_{\star}\right)$ for the case of fast rotation is larger than the co-rotation radius $\left(2.7 R_{\star}\right.$, indicated by the dotted line $)$.

\subsection{The emission measure}

The bulk of the X-ray emission in the class of late-type stars which we are aiming to model will originate from the closed flux region. The previous calculation allows us to determine an emission measure proxy from our model, which we define as

$E M \propto \int_{c} \rho^{2} \mathrm{~d} V$

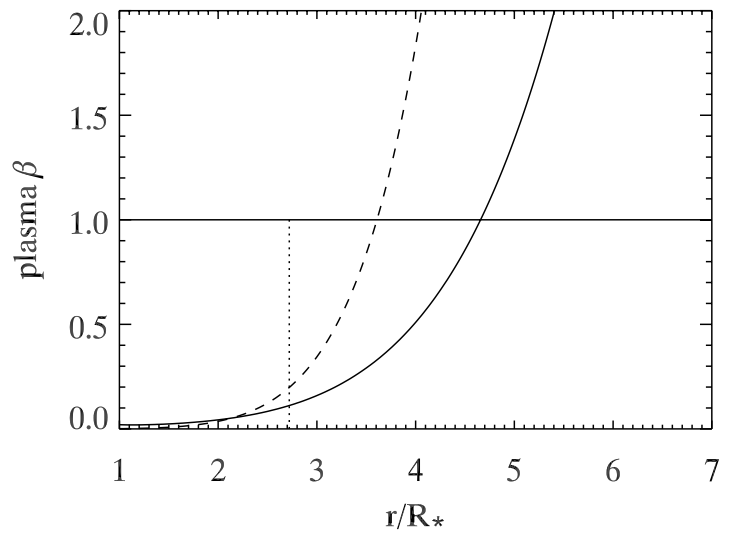

Fig. 1. Plot of the radial dependence of the model plasma $\beta$ in the equatorial plane $(\theta=\pi / 2)$ for two different angular velocities. The solid line shows the case of a star rotating with $\Omega=\Omega_{\odot}$ (slow rotation), the dashed line the case of a star with $\Omega=50 \Omega_{\odot}$ (fast rotation). The cutoff radii used for the model are given by the intersections of the plasma $\beta$ curves with the horizontal line $\beta=1$.0. The cut-off radius becomes smaller for increasing rotation rate due the growing importance of the centrifugal term. The co-rotation radius for the slow rotation case is at $37 R_{\star}$, outside the plotting region. The co-rotation radius of the fast rotation case is at $2.7 R_{\star}$, indicated by the dotted vertical line. For this set of parameters $\left(\beta_{\odot}=0.02, m=0.0, n=0.75, q=0.75, \xi_{3}=1.0\right.$, $R_{\star}=R_{\odot}, M_{\star}=M_{\odot}$ ) the cut-off radius is actually larger than the co-rotation radius.

with the integration volume being the closed field line region of the corona. The constant of proportionality will be fixed by setting the emission measure proxy for stars of solar rotation rate $\Omega=\Omega_{\odot}$ equal to the observed emission measure for stars of that rotation rate (see discussion in Sect. 3 below).

Given $r_{\mathrm{c}}$ we can calculate the emission measure proxy which takes the form

$E M \propto \rho_{\odot}^{2}\left(\frac{\Omega}{\Omega_{\odot}}\right)^{2 m} \int_{r=1}^{r_{\mathrm{c}}} \widehat{E M} r^{2} \mathrm{~d} r$

with the full form of $\widehat{E M}$ given by

$$
\begin{aligned}
\widehat{E M}= & \frac{\sqrt{\pi}}{2 \sqrt{\frac{2 \xi_{3}}{r}+\xi_{2} r^{2}}} \\
& \times \exp \left[\left(2 \xi_{1}+2 \xi_{3}\right)\left(\frac{1}{r}-1\right)++\xi_{2}\left(r^{2}-1\right)\right] \\
& \times \operatorname{erf}\left[\sqrt{\left(\frac{2 \xi_{3}}{r}+\xi_{2} r^{2}\right)\left(1-\frac{r}{r_{\mathrm{c}}}\right)}\right],
\end{aligned}
$$

where $\operatorname{erf}(x)$ is the error function (Abramowitz \& Stegun 1965). Details of the calculation are given in Appendix B.

Given the fundamental (solar) parameters $\left(T_{\odot}, \beta_{\odot}, \Omega_{\odot}\right.$, $R_{\star} / R_{\odot}, M_{\star} / M_{\odot}$ ) we must now make appropriate choices for the model parameter $\xi_{3}$, the plasma density, temperature and magnetic field scaling parameters $m, n, q$ and then calculate $\xi_{1}$ and $\xi_{2}$ at a given rotation rate, $\Omega$, from Eqs. (15) and (16) respectively. In the present paper we have taken $R_{\star} / R_{\odot}=1$, $M_{\star} / M_{\odot}=1, T_{\odot}=2 \times 10^{6} \mathrm{~K}$ and the solar rotation period to be 26 days. 
The influence of rotation on the density and temperature of stellar coronae has been highlighted by the recent results from Chandra and XMM/Newton (see Güdel et al. 2001; and Sanz-Forcada et al. 2003a, for XMM-Newton and EUVE results for a range of stars). For the Sun, the distribution of the emission measure with temperature shows a clear peak at around $10^{6} \mathrm{~K}$, while the more rapidly-rotating stars show an additional peak at around $10^{7} \mathrm{~K}$. Emission at this temperature is generally only seen on the Sun during flares (Drake et al. 2000). It appears that the more rapidly rotating stars show loops at a range of temperatures and with a corresponding range of densities. For AB Dor, Sanz-Forcada et al. (2003b) derive densities of $10^{10.8} \mathrm{~cm}^{-3}$ at $T=10^{6.3} \mathrm{~K}$ and $10^{12.5} \mathrm{~cm}^{-3}$ at $T=10^{7} \mathrm{~K}$. High densities for this star were also found by Maggio et al. (2000) and Güdel et al. (2001).

Our assumption of an isothermal plasma allows us to determine the emission measure for a corona composed entirely of loops at one temperature. Our choices of the scaling parameters must therefore try to encompass the range of observed values, bearing in mind that although we can scale the base density to the rotation rate in a simple way, the density in the corona depends also on the scale height, which is a function of temperature. The density that would be derived observationally from such a corona would also be weighted by the emission measure of each region and may well be a steeper function of rotation rate than that chosen for the base density.

We therefore chose fairly conservative limits on the scaling parameters. The observations mentioned above and observations of F, G and K dwarfs (e.g. Preibisch 1997; Güdel et al. 1997; Brickhouse \& Dupree 1998) suggest that a choice of $0<n<2$ would be appropriate for our temperature scaling parameter, although there remains some disagreement on the most appropriate method of deriving temperatures from fits to X-ray spectra (see Sanz-Forcada et al. 2002). For the magnetic field scaling parameter, observations (e.g. Montesinos \& Jordan 1993; Baliunas et al. 1996) suggest that a choice of $0<q<1$ may be appropriate. For the variation of plasma density with rotation a likely range should be $-1<m<1$ (see Maggio et al. 2000; Güdel et al. 2001; Brickhouse \& Dupree 1998). Similarly, the coronal base plasma $\beta$ should be taken from the range $0.01<\beta<0.3$.

The position of $r_{\mathrm{c}}$ will then give the volume of the closed field corona, over which we integrate the emission measure proxy. This procedure is repeated for a range of rotational velocities to allow us to construct the variation of emission measure with rotation and thereby allow comparison of our model results with observations such as those taken by Hempelmann et al. (1995).

\section{Results}

Since the parameters of our model are not completely determined by theory or observations we have investigated the dependence of our results on the variation of the parameters. It turns out that the results of the model calculations are relatively robust with respect to parameter variations.

Figures 2 to 6 show some of our results for certain illustrative model parameters. These results are compared with the

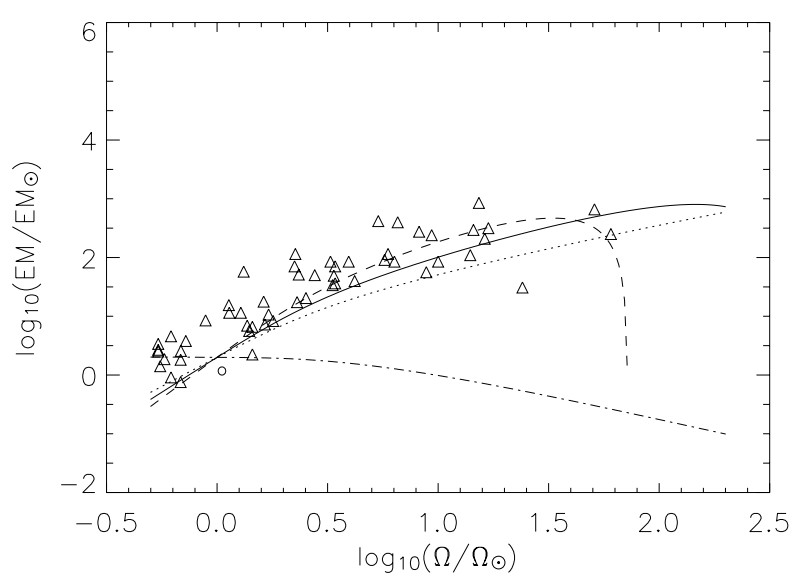

Fig. 2. Emission measure vs. angular velocity. The triangles show data taken from Hempelmann et al. (1995). The solid line represents the results of the reference model $\left(m=0.7, n=1.0, q=0.6, \beta_{\odot}=\right.$ 0.05 , and $\left.\xi_{3}=1\right)$. The position of the Sun is marked by a circle. The dash-dotted, dotted and dashed lines show the results of calculations with $m=-0.5, m=0.5$ and $m=0.9$, respectively, while all other parameter values are kept fixed. As expected the slope of the model curves increases with increasing $m$. The curve for negative $m$ clearly does not fit the data, whereas all the other curves are relatively close to each other.

observations of Hempelmann et al. (1995) which demonstrate saturation of $\mathrm{X}$-ray emission with rotation in main sequence $\mathrm{F}$ to $\mathrm{M}$ stars. Our choice of parameterisation gives us a certain degree of flexibility, but we must make sure that our choice of parameters lie within the ranges of possible values imposed by the observations.

In each diagram, the Sun is marked by an open circle. By comparison with the data of stars with similar rotation rates, it can be seen that the Sun does not have a typical X-ray luminosity for its rotation rate, but seems to be underluminous. We have therefore chosen to normalise the emission measure proxy at $\Omega / \Omega_{\odot}=1$ to twice the value of the solar emission measure since this seems to be a better representation of a typical latetype star at that rotation rate.

In each of the diagrams the solid curve corresponds to the parameter set $m=0.7, n=1.0, q=0.5, \beta_{\odot}=0.05$ and $\xi_{3}=1$. This parameter set serves as a reference case since it gives results which compare favourably with the observations. The other curves in each diagram show the results for parameter sets in which one of the parameters has been varied with respect to the reference set. This allows us to demonstrate the effect of the parameter variation on the result.

We remark that another possibility would be to match the model results to the upper envelope of the data, where the saturation effect seems to be more pronounced. However, it appears that the results would not change our main conclusions dramatically.

Figure 2 shows the effect of varying the exponent of the density scaling $(m)$. Shown are results for $m=-0.5$ (dashdotted line), $m=0.5$ (dotted line) and $m=0.9$ (dashed line). It can be clearly seen that the slope of the curves increases with increasing $m$. This is to be expected since the parameter $m$ directly affects the emission measure proxy through the factor 


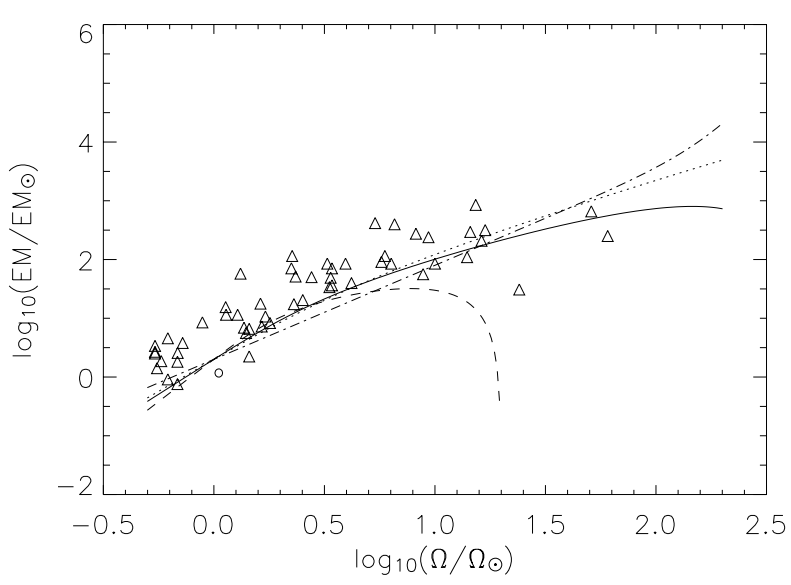

Fig. 3. Emission measure vs. angular velocity. The data (triangles) are the same as in Fig. 2. The solid line shows the reference case, the dash-dotted line to $n=0.2$, the dotted line corresponds to $n=0.8$ and the dashed line to $n=1.5$. The curve for the $n=0.2$ is almost a straight line for lower rotation rates, showing no saturation but an increase in emission measure for large rotation rates. It underestimates the emission measure for intermediate rotation rates. For increasing $n$ the saturation becomes more pronounced ( $n=0.8$ and $n=1.0$ ), but if $n$ becomes to large (see the dashed curve for $n=1.5$ ) a strong drop in emission measure occurs (here at about $10 \Omega_{\odot}$ ).

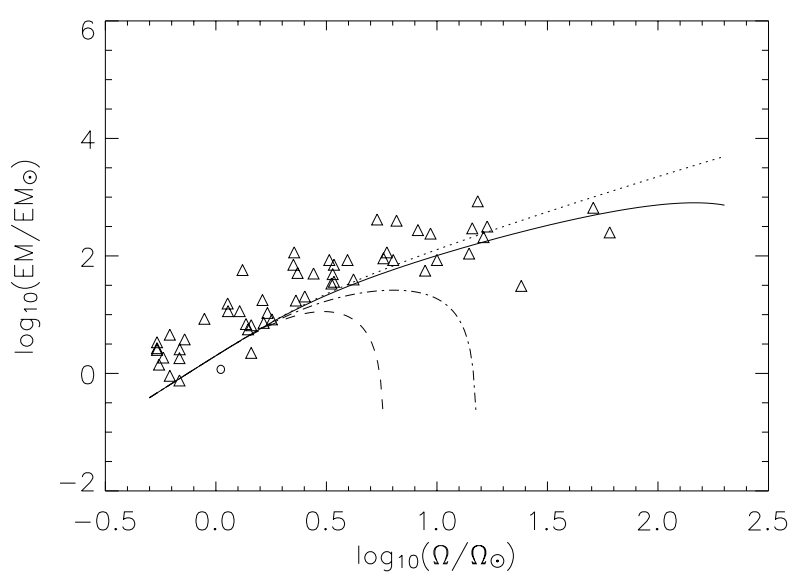

Fig. 4. Emission measure vs. angular velocity. The triangles show data taken from Hempelmann et al. (1995). The Sun is indicated by a circle. The solid line corresponds to the reference case, the dashed line to $q=0.0$, the dash-dotted line to $q=0.3$ and the dotted line to $q=0.7$. The plots show that the results are very similar for rotation rates below about $3 \Omega_{\odot}$, but that for larger angular velocities a larger value of the dynamo scaling parameter $q$ leads to a larger emission measure. The reason for this is that a larger $q$ implies a stronger magnetic field for a given angular velocity. This in turn implies that the closed flux region can be larger and more plasma can be confined by the field.

$\left(\Omega / \Omega_{\odot}\right)^{2 m}$ as can be seen from Eq. (29). The density scaling parameter $m$ also enters the results implicitly through the scaling of the coronal base plasma $\beta$, Eq. (27). The effect of this dependence of the emission measure proxy on $m$ is especially evident for the cases $m=-0.5$ and $m=0.9$. The direct dependence of the emission measure upon $\left(\Omega / \Omega_{\odot}\right)^{2 m}$ predicts a negative slope for $m=-0.5$, but this is obviously modulated by the implicit dependence of the cut-off radius on $m$ because the slope changes with changing rotation rate. For $m=-0.5$,

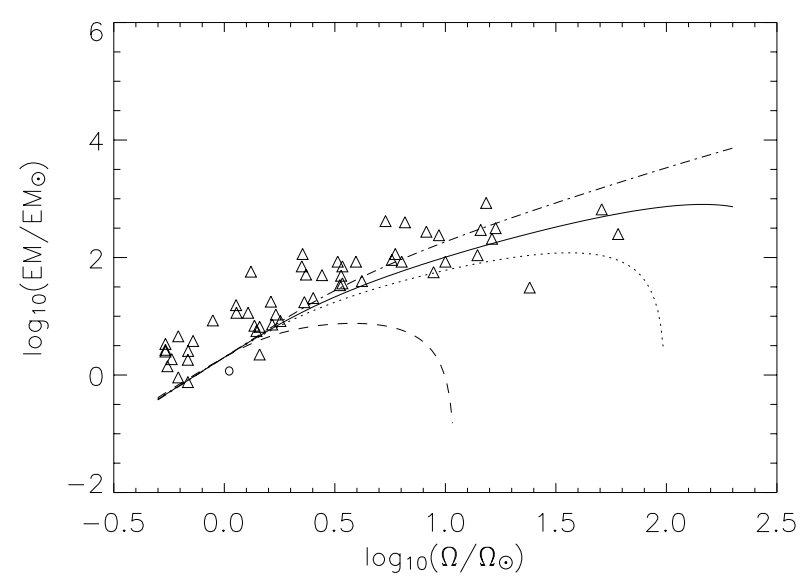

Fig. 5. Emission measure vs. angular velocity. The triangles show data taken from Hempelmann et al. (1995), the Sun is indicated by a circle. The reference case is plotted by the solid line. The dash-dotted line shows a case with $\beta_{\odot}=0.01$, the dotted line a case with $\beta_{\odot}=0.1$ and the dashed line a case with $\beta_{\odot}=0.3$. It is evident that for rotation rates larger than about $\Omega_{\odot}$ a larger $\beta_{\odot}$ leads to a smaller emission measure. This is due to the fact that a larger pressure will lead to a smaller cutoff radius and thus to a smaller closed flux region. Large values of $\beta_{\odot}$ (dashed line) lead to a drop in the emission measure already for small rotation rates $\left(\Omega \simeq 2 \Omega_{\odot}\right)$.

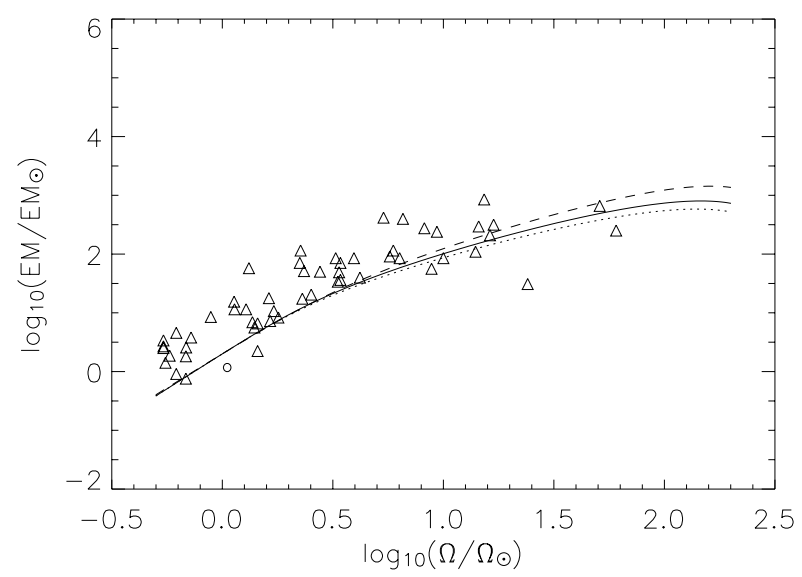

Fig. 6. Emission measure vs. angular velocity. The triangles show data taken from Hempelmann et al. (1995), the Sun is indicated by a circle. The reference case is plotted by the solid line. The dotted line shows results for $\xi_{3}=0.5$, the dashed line for $\xi_{3}=2.0$. Whereas the three lines are very hard to distinguish for rotation rates smaller than roughly $5 \Omega_{\odot}$, one can see that a larger value of $\xi_{3}$ implies a larger emission measure for faster rotators.

$n=1.0$ and $q=0.7$ the coronal base plasma $\beta$ decreases with increasing rotation rate which results in a larger extent of the closed flux region. This seems to partially compensate the decrease of the emission measure by the factor $\left(\Omega / \Omega_{\odot}\right)^{2 m}$ for small rotation rates, since the slope of the $m=-0.5$ curve is close to zero for $\log \left(\Omega / \Omega_{\odot}\right)<0.5$. The $m=-0.5$ curve does not fit the data at all, whereas the other three curves all give reasonable fits to the data for $\log \left(\Omega / \Omega_{\odot}\right)<1.5$. The effect of a strongly decreasing cut-off radius resulting in a strongly decreasing emitting volume can be seen for the dashed curve $(m=0.9)$. The larger value for $m$ leads to stronger increase in the coronal plasma density and pressure which in turn leads to 
the rapid decrease of the cut-off radius and hence of the emission measure for large rotation rates.

In Fig. 3 the effect of varying the temperature scaling parameter $n$ are shown. Again the solid line shows the reference model. The dash-dotted line shows results for $n=0.2$, the dotted line for $n=0.8$ and the dashed line for $n=1.5$. The parameter $n$ enters into the calculation of the cut-off radius through $\xi_{1}$, $\xi_{2}$ and the coronal base plasma $\beta$.

A smaller value of $n$ has the same effect on the coronal base plasma $\beta$ as a smaller value of the density scaling parameter $m$. For $m=0.7, n=0.2$ and $q=0.5$, the coronal base plasma $\beta$ actually decreases with increasing rotation velocity. This has the effect that the cut-off radii for this value of $n$ will be larger than the cut-off radii obtained with a larger $n$. This can be clearly seen in Fig. 3, the $n=0.2$ curve is nearly a straight line for all rotation rates below $\log \left(\Omega / \Omega_{\odot}\right) \approx 1.5$. Above this rotation rate the curve actually shows an increasing slope, clearly not as observed. For increasing $n$ the saturation effect due to a stronger decrease of the emitting volume becomes more and more obvious, and for $n=1.5$ one gets a strong drop in the emission measure for rotation rates larger than roughly $10 \Omega_{\odot}$.

Although the influence of $n$ on the coronal base plasma $\beta$ is probably the most important one for larger rotation rates, $n$ obviously also has an influence for smaller rotation rates through the parameters $\xi_{1}$ and $\xi_{2}$. The dimensionless parameter $\xi_{1}$ is a measure of the importance of the gravitational energy of a plasma particle in the corona compared to its thermal energy. For a given rotation rate a decrease in $n$ means an increase in $\xi_{1}$ (for $\Omega>\Omega_{\odot}$ ). However, the importance of the gravitational term generally decreases for larger rotation rates compared to the centrifugal term. The parameter $\xi_{2}$ measures the importance of the energy associated with the rotation of the plasma compared to the thermal energy. It seems that for intermediate rotation rates $\left(\Omega\right.$ slightly larger than $\Omega_{\odot}$ ) the emission measure values increase with increasing $n$, but it is not exactly clear what the reason for this is as both $\xi_{1}$ and $\xi_{2}$ enter both the expression for the emission measure and the equation determining the cutoff radius in a strongly nonlinear way.

Figure 4 shows the effect of varying the dynamo scaling parameter $q$. Again the solid line shows the reference model $(q=0.6)$. The dashed line shows the results for $q=0.0$, the dash-dotted line for $q=0.3$, and the dotted line for $q=0.7$. Comparing the solid line with the other lines it is evident that a higher $q$ leads to a higher emission measure. The reason is that for a larger value of $q$ the magnetic field strength grows faster with rotation rate and therefore more plasma can be confined by the magnetic field. Within our model, this effect can be seen in Eq. (27) where an increasing $q$ leads to a slower increase of the surface plasma $\beta$. This in turn affects the cut-off radius, and thus the emitting volume. For the smaller $q$ values one can actually see that if the surface magnetic field does not increase with rotation rate (dashed line) or increases too slowly (dash-dotted line), the emission measure (super-)saturates far too early. This is due to a drop in the emitting volume caused by the decrease of the cut-off radius.

The effect of varying the coronal base plasma $\beta_{\odot}$ is shown in Fig. 5. We show results for $\beta_{\odot}=0.01$ (dash-dotted line), $\beta_{\odot}=0.1$ (dotted line) and $\beta_{\odot}=0.3$ (dashed line) in comparison with the reference case $\left(\beta_{\odot}=0.05\right.$, solid line). An increase of $\beta_{\odot}$ leads to lower values of the emission measure because a larger $\beta_{\odot}$ leads to a smaller cut-off radius. This can be seen from Eq. (26). An increase in $\beta_{\odot}$ increases the relative importance of plasma pressure compared to magnetic pressure and therefore decreases the cut-off radius. This is most obvious for the case with $\beta_{\odot}=0.3$, in which the emission measure drops already for rotation rates of approximately $2 \Omega_{\odot}$. A similar drop, but for larger rotation rates can be seen for the $\beta_{\odot}=0.1$ case (dotted line).

Finally, we show the effect of different $\xi_{3}$ values in Fig. 6. Apart from the reference case $\left(\xi_{3}=1.0\right.$, solid line), we show result for $\xi_{3}=0.5$ (dotted line) and $\xi_{3}=2.0$ (dashed line). For rotation rates smaller than about $5 \Omega_{\odot}$ the three curves are nearly identical. For rotation rates larger than $5 \Omega_{\odot}$ the emission measure increases with increasing $\xi_{3}$. This behaviour can be explained by looking at Eq. (26). The parameter $\xi_{1}$ decreases as the rotation rate increases, whereas $\xi_{3}$ is constant. The term containing $\xi_{3}$ in the argument of the exponential function is negative $\left(r_{\mathrm{c}}>1\right)$, and therefore a larger value of $\xi_{3}$ implies a smaller value of this part of the exponential function and thus a larger cut-off radius, which in turn results in a larger emission measure.

\section{Discussion and conclusions}

In the present paper we have suggested a simple model which is able to explain the observed saturation and super-saturation effects in the X-ray emission of late-type stars without the need to invoke e.g. a nonlinear saturation mechanism for the stellar dynamo. The model is based on a number of assumptions, namely

1. the stellar magnetic field is a dipole field with the dipole axis aligned with the rotation axis;

2. the plasma in the stellar corona is an isothermal ideal gas;

3. the bulk of the X-ray emission originates from the closed flux region of the corona;

4. the amount of closed magnetic flux for a given rotation rate can be determined by a heuristic criterion based on the ratio between the plasma pressure and the magnetic pressure;

5. the basic coronal parameters depend on the stellar angular velocity via power laws.

These assumptions allowed us to calculate the emission measure as a function of rotation rate for a given set of parameters. For larger rotation rates the amount of closed flux in the stellar corona/magnetosphere decreases and therefore the X-ray emitting volume becomes smaller. This in turn can lead to a saturation (or even super-saturation) when the increase in density in the closed flux region is compensated (or over-compensated) by a decrease of the emitting volume. This is very similar in spirit although not in terms of the physics to the coronal stripping model proposed by Jardine \& Unruh (1999).

The results compare well with observations made e.g. by Hempelmann et al. (1995). We also demonstrated that the model is relatively robust with respect to small variations of the model parameters. This is important because most of the 


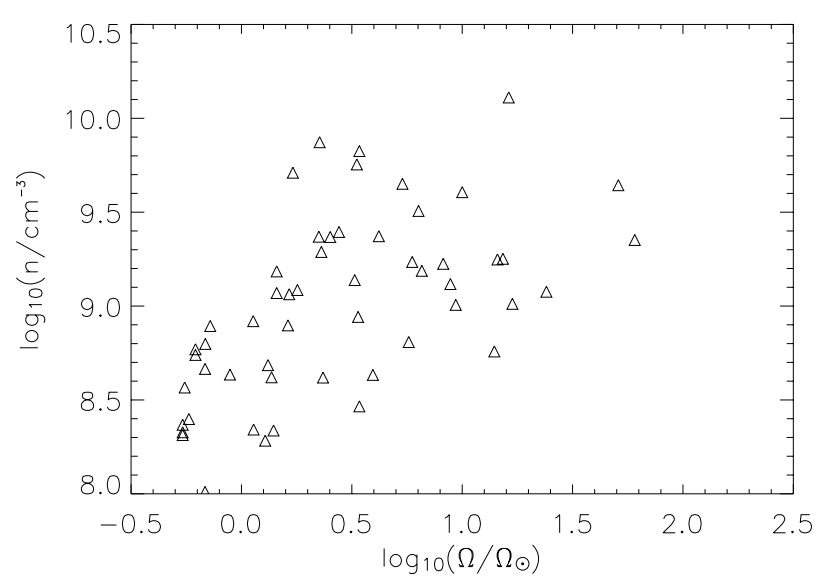

Fig. 7. Average electron densities plotted against angular velocity. The densities have been derived using Eq. (32), taking the emission measure data from Hempelmann et al. (1995). The emitting volumes have been taken from the standard parameter set used in Figs. 2 to 6 . The values of the average density are lower than those found by e.g. Ness et al. (2003).

parameter values are not constrained very much by either theory or observations.

A further comparison of our model with observational results by Chandra and XMM-Newton (e.g. Ness et al. 2003) is possible if one combines the observed emission measures taken from Hempelmann et al. (1995) and the emitting volume $\left(V_{\mathrm{emit}}\right)$ from our model for a given set of parameters and rotation rate to estimate the average electron density $\left(\bar{n}_{\mathrm{e}}\right)$ from

$E M \approx \bar{n}_{\mathrm{e}}^{2} \cdot V_{\text {emit }}$.

In our normalisation we obtain

$\bar{n}_{\mathrm{e}}=9.685 \times 10^{8}\left(\frac{E M}{E M_{\odot}}\right)^{\frac{1}{2}}\left(\frac{V_{\mathrm{emit}}}{R_{\star}^{3}}\right)^{-\frac{1}{2}} \mathrm{~cm}^{-3}$

where we have used the active Sun value $E M_{\odot} \approx 10^{50.5} \mathrm{~cm}^{-3}$ (e.g. Golub \& Pasachoff 1997, p. 104). In the model the emitting volume is the volume enclosed between the stellar surface and the dipole field line crossing the equatorial plane at the cutoff radius $r_{\mathrm{c}}$. A straightforward calculation shows that

$V_{\mathrm{emit}}=\frac{8 \pi}{105}\left(8 r_{\mathrm{c}}^{5}+4 r_{\mathrm{c}}^{2}+3 r_{\mathrm{c}}-15\right) \sqrt{1-\frac{1}{r_{\mathrm{c}}}}$.

A plot of average densities vs. angular velocity determined by Eq. (32) is shown in Fig. 7. The emitting volumes used in this plot have been calculated from the standard model parameter set also used in Figs. 2 to 6 . We find that the average densities derived by this method are roughly between $\log \left(n_{\mathrm{e}} / \mathrm{cm}^{-3}\right) \approx$ 8.0-10.0. There is a general trend to higher average densities with increasing angular velocity. These average densities are about one to two orders of magnitude lower than the range of densities found e.g. by Ness et al. (2003) for stars with magnetically active coronae $\left(\log \left(n_{\mathrm{e}} / \mathrm{cm}^{-3}\right) \approx 10.0-11.0\right)$ and another order of magnitude lower than the values found for AB Dor by Sanz-Forcada et al. (2003b) for the high-temperature part of the corona. The difference between the average densities derived from the model and the observed densities is, however, not unexpected. First of all the average density is not necessarily a good representation of the values of the plasma density in our model. The model allows the density to vary both along and across field lines, and the variation can be larger than two orders of magnitude depending on the rotation rate and the model parameters. For example, the value of the average density for a star with solar rotation rate and solar emission measure calculated using this method is $\bar{n}_{\mathrm{e}}=9.7 \times 10^{7} \mathrm{~cm}^{-3}$, a value which is about two orders of magnitude lower than the actual value of the average density of the solar corona. This is a typical case in which the model density shows a strong variation with radius which leads to a significant underestimation of the density by the procedure used above. Another way of looking at the same problem is that in the calculations presented above we take the emitting volume to be the complete closed flux volume defined by our model whereas in most cases only a part of this volume contributes noticeably to the emission measure proxy (similar to a filling factor smaller than one). We conclude that the model does systematically underestimate the average electron densities (if determined as in Eq. (32)), but that this is partially due to the relative simplicity of the present model which certainly contributes to its robustness, but also implies that there is room for improvements.

One possible improvement would be a more sophisticated treatment of the stellar magnetic field, for which there are various possibilities. In the present model we are using the simplest possible magnetic field model, a dipole field. One could think of introducing more complicated magnetic fields by e.g. adding higher multipoles. Such a magnetic field would have more small scale structure close to the star, but the higher multipoles drop off faster than the dipole field with growing distance from the stellar surface. Additional structure could be especially important for slow rotators $\left(\Omega \sim \Omega_{\odot}\right)$ since we know that the solar $\mathrm{X}$-ray emission does not really originate in a large-scale dipolelike magnetic field region, but in many small-scale loops. This could mean that assuming a global dipole may lead to an overestimate of the emission, especially in the slow rotator regime. On the other hand the Sun seems to be underluminous compared with stars of the same rotation rate and therefore might be untypical. A practical difficulty would be to find the correct boundary conditions for the higher order multipole contributions, i.e. their relative strength compared to the dipole component. It is not clear at the moment how this problem could be solved. Also for more complicated magnetic fields the implementation of the cut-off criterion for closed flux would be much more difficult (a more detailed discussion of the modelling of the structure and magnetic topology of rapidly rotating stellar corona may be found in Hussain et al. 2002).

Another possible improvement of the model would be the change to boundary conditions in such a way that the open magnetic flux is really open. For example, staying within the framework of a current free model one could think of solutions which have an inner closed field line region and an outer region in which field lines do not cross the equatorial plane, and become e.g. approximately radial for large distances from the star. The mathematical difficulties of such a model would, however, be much bigger than in the present model, and it is not clear 
whether the improvements would outweigh the increase in difficulty.

We are presently investigating how deviations from the current free assumption for the magnetic field change the results of the model. Especially for rapid rotators the plasma in the outer regions of the closed magnetic field lines extending beyond the co-rotation radius will exert an outward centrifugal force which can only be compensated by an inward Lorentz force caused by a stretching of the magnetic field lines. This means that the nonlinear partial differential Eq. (8) has to be solved, which can only be done numerically. The results of this investigation should give us an indication how well the assumption of a current free magnetic field works.

Further improvements could be made by dropping the assumption of an isothermal atmosphere and by coupling Eqs. (8) and (9) with an energy equation. This would provide us with the possibility of unifying the model of Jardine \& Unruh (1999) with the present model, but would also lead to a more complicated model. Such a model would be much harder to investigate, but it could provide further confirmation of the coronal stripping mechanism.

We would like to emphasize that we see the mechanism proposed in this paper not as being the only possible mechanism causing the observed phenomena in stellar coronae, nor do we assert that there is no saturation of the dynamo mechanism. What we have sought to demonstrate is that there are other mechanisms which may mimic this phenomenon and mask the rotation velocity at which saturation will appear. We believe that it is highly likely that a combination of different mechanisms is operating at the same time, and further theoretical and observational investigations will be needed to decide which of the proposed mechanisms are actually at work.

Acknowledgements. We thank Andrew Collier Cameron for useful discussions. We would also like to thank the anonymous referee whose remarks have helped to improve the paper. This work has been supported by PPARC through a Ph.D. studentship (Richard Ryan) and an Advanced Fellowship (Thomas Neukirch). Richard Ryan and Thomas Neukirch also acknowledge financial support provided through the European Community's Human Potential Programme under contract HPRN-CT-2000-00153, PLATON.

\section{Appendix A: Motivation of exponential pressure function by Vlasov approach}

MHD does not provide us with a means to deduce the function $p_{0}(A)$. We therefore motivate our choice of an exponential dependence of $p_{0}$ upon $A$ by a calculation using Vlasov theory. We emphasize that this is not intended to be a rigorous derivation of $p_{0}(A)$, but more a guideline for a sensible choice of that function.

We follow the work of Neukirch (1993) who has used a Vlasov approach to investigate equilibria of collisionless rigidly rotating magnetospheres of planets. Neukirch (1993) does not include gravity in his calculation and uses a different class of distribution functions, but we can make use of some of his general results.

To find Vlasov equilibria one has to specify stationary solutions of the Vlasov equations, i.e. equilibrium distribution functions $f_{s}$ for each particle species $s$. The equilibrium solutions of the Vlasov equation generally depend on the constants of motion of the system. Using spherical coordinates $r, \theta, \phi$ and velocity components $v_{r}, v_{\theta}$ and $v_{\phi}$, two constants of motion of a conservative axisymmetric system as in our case are the one-particle energy (Hamiltonian)

$H=\frac{1}{2} m_{s} v^{2}+q_{s} \Phi+m_{s} \psi$,

and the canonical momentum associated with the azimuthal angle $\phi$,

$p_{\phi}=m_{s} r \sin \theta v_{\phi}+q_{s} A$.

Here, $m_{s}$ is the mass of a particle of species $s, q_{s}$ its electric charge, $\Phi$ is the electric potential, $\psi=-G M_{\star} / r$ the gravitational potential and $A$ the flux function. Apart from the gravitational potential the expressions (A.1) and (A.2) are identical with those of Neukirch (1993).

For simplicity we now assume that we only have two particles species with opposite electric charge of equal magnitude (e.g. electrons and protons). The crucial assumption is that the distribution function of each particle species has the form of a drifting Maxwell-Boltzmann distribution (i.e. a shifted Gaussian in the three velocity components) in velocity space, with drift in the $\phi$ direction. We also assume for convenience that both species have the same temperature. This is not essential, but facilitates the further calculations. With these assumptions we can write the distribution function in the form

$f_{s}=f_{0 s} \exp \left[-\beta\left(H-\Omega_{s} p_{\phi}\right)\right]$,

where $\beta=1 /\left(k_{\mathrm{B}} T\right), f_{s 0}$ is a normalisation constant and $\Omega_{s}$ is a constant real parameter, which turns out to be the average angular velocity of species $s$.

Using these distribution functions all other quantities like density and pressure can be calculated, using the appropriate velocity moments of the distribution functions. Here, we only need the particle density and the plasma pressure. The particle density is needed to eliminate the electric potential $\Phi$ by using the assumption of a quasi-neutral plasma $\left(n_{\mathrm{e}}=n_{\mathrm{i}}\right)$. The plasma pressure will generally be a tensor in Vlasov theory, but one can show (e.g. Neukirch 1993) that for distribution functions of the type (A.3) the pressure is indeed a scalar.

The velocity moments are more easily calculated if we introduce two new quantities, namely

$\tilde{v}_{\phi}=v_{\phi}-\Omega_{s} r \sin \theta$

and

$\tilde{H}=H-\Omega_{s} p_{\phi}=\frac{1}{2} m_{s}\left(v_{r}^{2}+v_{\theta}^{2}+\tilde{v_{\phi}}\right)^{2}-\tilde{H}_{\min }$,

where

$\tilde{H}_{\min }=q_{s}\left(\Phi-\Omega_{s} A\right)-\frac{1}{2} m_{s}\left(\Omega^{2} r^{2} \sin ^{2} \theta\right)+m_{s} \psi$.

The new quantities $\tilde{v}_{\phi}$ and $\tilde{H}$ are the $\phi$ component of the velocity and the Hamiltonian in a frame of reference rotating with angular velocity $\Omega_{s}$ with respect to the inertial frame. $\tilde{H}_{\text {min }}$ is the minimum value of this Hamiltonian at any given point in 
configuration space. The particle density of each species may now be found by integrating the distribution function over velocity space. Following Neukirch (1993) we have

$$
\begin{aligned}
n_{s} & =\int_{-\infty}^{\infty} f_{s}(\tilde{H}) \mathrm{d}^{3} v \\
& =\frac{4 \sqrt{2} \pi}{\sqrt{m_{s}}} f_{0 s} \int_{\tilde{H}_{\min }}^{\infty} \sqrt{\tilde{H}-\tilde{H}_{\min }} \exp (-\beta \tilde{H}) \mathrm{d} \tilde{H} .
\end{aligned}
$$

Equation (A.7) can be easily evaluated by using $\beta\left(\tilde{H}-\tilde{H}_{\min }\right)$ as a new variable. The final expression for the density is

$$
\begin{aligned}
n_{s}=n_{0 s} \exp [-\beta & \left\{q_{s}\left(\Phi-\Omega_{s} A\right)\right. \\
& \left.\left.-\frac{1}{2} m_{s} \Omega_{s}^{2} r^{2} \sin ^{2} \theta+m_{s} \psi\right\}\right]
\end{aligned}
$$

where $n_{0 s}=4 \sqrt{2}{\sqrt{\pi /\left(\beta m_{s}\right)}}^{3} f_{0 s}$.

As usual we now eliminate the electric potential $\Phi$ by assuming that the plasma is quasi-neutral, i.e. $n_{\mathrm{e}}=n_{\mathrm{i}}=n$. Using Eq. (A.8) and assuming without loss of generality that $n_{0 e}=n_{0 i}$ we obtain

$e \Phi=e \frac{\Omega_{\mathrm{i}}+\Omega_{\mathrm{e}}}{2} A+\frac{1}{4}\left(m_{\mathrm{i}} \Omega_{\mathrm{i}}^{2}-m_{\mathrm{e}} \Omega_{\mathrm{e}}^{2}\right) r^{2} \sin ^{2} \theta-\frac{m_{\mathrm{i}}-m_{\mathrm{e}}}{2} \psi$.

Substituting this expression for $\Phi$ back into either $n_{\mathrm{i}}$ or $n_{\mathrm{e}}$ we get for the quasi-neutral particle density

$n=n_{0} \exp \left(A / A_{0}\right) \exp \left(-U_{\text {kin }} / k_{\mathrm{B}} T\right)$

with

$A_{0}=\frac{2 k_{\mathrm{B}} T}{e\left(\Omega_{\mathrm{i}}+\Omega_{\mathrm{e}}\right)}$

and

$U_{\text {kin }}=-\frac{1}{2}\left(m_{\mathrm{i}} \Omega_{\mathrm{i}}^{2}+m_{\mathrm{e}} \Omega_{\mathrm{e}}^{2}\right) r^{2} \sin ^{2} \theta+\frac{m_{\mathrm{i}}+m_{\mathrm{e}}}{2} \psi$.

Here $U_{\text {kin }}$ is the kinetic equivalent of the $U$ defined in Eq. (5) and actually reduces to that expression in the MHD limit.

The pressure is defined by the velocity moment (e.g. Neukirch 1993)

$p=\sum_{s} \frac{m_{s}}{3} \int_{-\infty}^{\infty}\left(v_{r}^{2}+v_{\theta}^{2}+\tilde{v}_{\phi}^{2}\right) f_{s} \mathrm{~d}^{3} v$.

Using the same substitutions as used in calculating the density and the quasi-neutral particle density, it is straightforward to show that $p=k_{\mathrm{B}} T n$ and thus

$p=k_{\mathrm{B}} T n_{0} \exp \left(A / A_{0}\right) \exp \left(-U_{\text {kin }} / k_{\mathrm{B}} T\right)$

which is exactly of the form used in our model.

\section{Appendix B: Derivation of emission measure proxy}

Once $r_{\mathrm{c}}$ and $A_{\mathrm{c}}$ have been determined, we can calculate the emission measure proxy using Eq. (28). The integral over $\rho^{2}$ can be reduced from an integral over $r$ and $\theta$ to an integral over $r$. Using the symmetry of our model with respect to the equatorial plane $(\theta=\pi / 2)$ we obtain

$\int_{V_{\mathrm{c}}} \rho^{2} \mathrm{~d} V=4 \pi \rho_{\star}^{2} \int_{r=1}^{r_{\mathrm{c}}}\left\{\int_{\theta=\theta_{\mathrm{c}}}^{\frac{\pi}{2}} F(r, \theta) \sin \theta \mathrm{d} \theta\right\} r^{2} \mathrm{~d} r$

with

$$
\begin{aligned}
& F(r, \theta)=\exp \left[2 \xi_{3}\left(\frac{\sin ^{2} \theta}{r}-1\right)\right. \\
& \left.+2 \xi_{1}\left(\frac{1}{r}-1\right)+\xi_{2}\left(r^{2} \sin ^{2} \theta-1\right)\right]
\end{aligned}
$$

where $\theta_{\mathrm{c}}=\theta_{\mathrm{c}}\left(r, r_{\mathrm{c}}\right)=\arcsin \left(\sqrt{r / r_{\mathrm{c}}}\right)$ and where $V_{\mathrm{c}}$ is the volume of the closed flux region of the magnetosphere. The $\theta$ integral can be carried out by introducing the new variable $\mu=\cos \theta$ for which the corresponding cut-off value would be $\mu_{\mathrm{c}}=\cos \theta_{\mathrm{c}}=\sqrt{1-\left(r / r_{\mathrm{c}}\right)}$. Rewriting $F(r, \theta)$ as

$$
\begin{array}{r}
F(r, \theta)=\exp [ \\
\left.\left[2 \xi_{1}+2 \xi_{3}\right)\left(\frac{1}{r}-1\right)+\xi_{2}\left(r^{2}-1\right)\right] \\
\times \exp \left[-\left(\xi_{2} r^{2}+2 \xi_{3} \frac{1}{r}\right) \cos ^{2} \theta\right]
\end{array}
$$

the $\theta$ integral then becomes

$$
\begin{aligned}
\widehat{E M}= & \exp \left[\left(2 \xi_{1}+2 \xi_{3}\right)\left(\frac{1}{r}-1\right)+\xi_{2}\left(r^{2}-1\right)\right] \\
& \times \int_{0}^{\mu_{c}} \exp \left[-\left(\xi_{2} r^{2}+2 \xi_{3} \frac{1}{r}\right) \mu^{2}\right] \mathrm{d} \mu .
\end{aligned}
$$

Using the substitution

$$
t=\sqrt{\frac{2 \xi_{3}}{r}+\xi_{2} r^{2}} \mu
$$

the integral may now be expressed as

$$
\begin{aligned}
\widehat{E M}= & \frac{1}{\sqrt{\frac{2 \xi_{3}}{r}+\xi_{2} r^{2}}} \\
& \times \exp \left[\left(2 \xi_{1}+2 \xi_{3}\right)\left(\frac{1}{r}-1\right)+\xi_{2}\left(r^{2}-1\right)\right] \\
& \times \int_{0}^{t_{c}} \exp \left(-t^{2}\right) \mathrm{d} t
\end{aligned}
$$

where

$t_{\mathrm{c}}=\sqrt{\left(\frac{2 \xi_{3}}{r}+\xi_{2} r^{2}\right)\left(1-\frac{r}{r_{\mathrm{c}}}\right)}$.

The integral over $t$ can be expressed as an error function (Abramowitz \& Stegun 1965) defined as

$\operatorname{erf}(x)=\frac{2}{\sqrt{\pi}} \int_{0}^{x} \exp \left(-t^{2}\right)$

The final form of the $\theta$ component of the integral is

$$
\begin{aligned}
\widehat{E M}= & \frac{\sqrt{\pi}}{2 \sqrt{\frac{2 \xi_{3}}{r}+\xi_{2} r^{2}}} \\
& \times \exp \left[\left(2 \xi_{1}+2 \xi_{3}\right)\left(\frac{1}{r}-1\right)+\xi_{2}\left(r^{2}-1\right)\right] \\
& \times \operatorname{erf}\left[\sqrt{\left(\frac{2 \xi_{3}}{r}+\xi_{2} r^{2}\right)\left(1-\frac{r}{r_{\mathrm{c}}}\right)}\right] .
\end{aligned}
$$




\section{References}

Abramowitz, M., \& Stegun, I. A. 1965, Handbook of Mathematical Functions (Dover: New York), 253

Baliunas, S., Sokoloff, D., \& Soon, W. 1996, ApJ, 457, L99

Brickhouse, N., \& Dupree, A. K. 1998, ApJ, 502, 918

Charbonneau, P., \& MacGregor, K. B. 1992, ApJ, 387, 639

Collier Cameron, A., Jianke, L., \& Mestel, L. 1991, in NATO ASI Series Angular Momentum Evolution of Young Stars, ed. S. Catalano, \& J. R. Stauffer, 297

Donati, J.-F., \& Collier Cameron, A. 1997, MNRAS, 291, 1

Drake, J. J., Peres, G., Orlando, S., Laming, J. M., \& Maggio, A. 2000, ApJ, 545, 1074

Durney, B. R. 1972, in Asilomar Conference on the Solar Wind, ed. C. P. Sonnett, P. J. Coleman Jr., \& J. M. Wilcox (Washington: NASA), 282

Golub, L., \& Pasachoff, J. M. 1997, The Solar Corona (Cambridge: Cambridge University Press)

Güdel, M., Guinan, E. F., \& Skinner, S. L. 1997, ApJ, 483, 947

Güdel, M., Audard, M., den Boggende, A. J., et al. 2001, in X-ray Astronomy 2000, ed. R. Giacconi, S. Serio, \& L. Stella, ASP Conf. Ser., 234, 73

Hempelmann, A., Schmidt, J. H. M. M., Schultz, M., Rüdiger, G., \& Stępień, K. 1995, A\&A, 294, 515

Hussain, G. A. J., van Ballegooijen, A. A, Jardine, M., \& Collier Cameron, A. 2002, ApJ, 575, 1078

Jardine, M. M., \& Unruh, Y. C. 1999, A\&A, 346, 883

Kraft, R. P. 1967, ApJ, 150, 551

Maggio, A., Pallavicini, R., Reale, F., \& Tagliaferri, G. 2000, A\&A, 356,627

Mathioudakis, M., Fruscione, A., Drake, J. J., et al. 1995, A\&A, 300, 775

Mestel, L., \& Spruit, H. C. 1987, MNRAS, 226, 57

Montesinos, B., \& Jordan, C. 1993, MNRAS, 264, 900

Ness, J.-U., Audard, M., Schmitt, J. H. M. M., \& Güdel, M. 2003, Adv. Space Res., 32, 937
Neukirch, T. 1993, J. Geophys. Res., 98, 3753

Noyes, R. W., Hartmann, L. W., Baliunas, S. L., Duncan, D. K., \& Vaughan, A. H. 1984, ApJ, 279, 763

O’Dell, M. A., Panagi, P. M., Hendry, M. A., \& Collier Cameron, A. 1995, A\&A, 294, 715

Parker, E. N. 1955, ApJ, 122, 293

Parker, E. N. 1979, Cosmical Electric Fields (England: Oxford University Press)

Pizzolato, N., Maggio, A., Micela, G., Sciortino, S., \& Ventura, P. 2003, A\&A, 397, 147

Preibisch, T. 1997, A\&A, 320, 525

Prosser, C. F., Randich, S., Stauffer, J. R., \& Schmidt, J. H. M. M. 1996, AJ112, 1570

Randich, S. 1998, in 10th Cambridge Workshop on Cool Stars, Stellar Systems and the Sun, ed. D. Donahue, \& J. Bookbinder, ASP Conf. Ser., 154, 501

Rosner, R. 2000, Phil. Trans. R. Soc. London, Ser. A, 358, 689

Saar, S. 2001, in 11th Cambridge Workshop on Cool Stars, Stellar Systems and the Sun, ed. R. J. García López, R. Rebolo, \& M. R. Zapatero Osorio, ASP Conf. Ser., 223, 295

Sanz-Forcada, J., Brickhouse, N.S., \& Dupree, A.K. 2002, ApJ, 570, 799

Sanz-Forcada, J., Brickhouse, N. S., \& Dupree, A. K. 2003a, ApJS, 145,147

Sanz-Forcada, J., Maggio, A., \& Micela, G. 2003b, A\&A, 408, 1087

Schüssler, M., \& Solanki, S. K. 1992, A\&A, 264, L13

Schüssler, M., Caligari, P., Ferriz-Mas, A., Solanki, S. K., \& Stix, M. 1996, A\&A, 314, 503

Solanki, S. K., Motamen, S., \& Keppens, R. 1997, A\&A, 325, 1039

Stauffer, J. R., Hartmann, L. W., Prosser, C. F., et al. 1997, ApJ, 479, 776

Vilhu, O. 1984, A\&A, 133, 117

Zombeck, M. V. 1990, Handbook of Space Astronomy and Astrophysics (England: Cambridge University Press) 